\title{
How to Complete a Doubling Metric
}

\author{
Anupam Gupta* $\quad$ Kunal Talwar ${ }^{\dagger}$
}

\begin{abstract}
In recent years, considerable advances have been made in the study of properties of metric spaces in terms of their doubling dimension. This line of research has not only enhanced our understanding of finite metrics, but has also resulted in many algorithmic applications. However, we still do not understand the interaction between various graph-theoretic (topological) properties of graphs, and the doubling (geometric) properties of the shortest-path metrics induced by them. For instance, the following natural question suggests itself: given a finite doubling metric $(V, d)$, is there always an unweighted graph $\left(V^{\prime}, E^{\prime}\right)$ with $V \subseteq V^{\prime}$ such that the shortest path metric $d^{\prime}$ on $V^{\prime}$ is still doubling, and which agrees with $d$ on $V$. This is often useful, given that unweighted graphs are often easier to reason about.

A first hurdle to answering this question is that subdividing edges can increase the doubling dimension unboundedly, and it is not difficult to show that the answer to the above question is negative. However, surprisingly, allowing a $(1+\varepsilon)$ distortion between $d$ and $d^{\prime}$ enables us bypass this impossibility: we show that for any metric space $(V, d)$, there is an unweighted graph $\left(V^{\prime}, E^{\prime}\right)$ with shortest-path metric $d^{\prime}: V^{\prime} \times V^{\prime} \rightarrow \mathbb{R}_{\geq 0}$ such that

- for all $x, y \in V$, the distances $d(x, y) \leq d^{\prime}(x, y) \leq(1+\varepsilon) \cdot d(x, y)$, and

- the doubling dimension for $d^{\prime}$ is not much more than that of $d$, where this change depends only on $\varepsilon$ and not on the size of the graph.

We show a similar result when both $(V, d)$ and $\left(V^{\prime}, E^{\prime}\right)$ are restricted to be trees: this gives a simple proof that doubling trees embed into constant dimensional Euclidean space with constant distortion. We also show that our results are tight in terms of the tradeoff between distortion and dimension blowup.
\end{abstract}

\footnotetext{
${ }^{*}$ Computer Science Department, Carnegie Mellon University, Pittsburgh, PA 15213. This research was partly supported by the NSF CAREER award CCF-0448095, and by an Alfred P. Sloan Fellowship.

${ }^{\dagger}$ Microsoft Research, Silicon Valley Campus, 1065 La Avenida, Mountain View, CA 94043.
} 


\section{Introduction}

The algorithmic study of finite metrics has become a central theme in theoretical computer science in recent years. Of particular interest has been the study of the geometry of metrics - embeddings into Minkowski spaces have been the most obvious example, accompanied by the study of notions of metric dimension which have allowed us to partially quantify geometric properties that make metrics tractable for several algorithmic problems.

Given these advances in our understanding of the geometric properties of abstract metric spaces, it is worth remarking that our comprehension of the topological properties of metric spaces - and of the relationship between topology and geometry has lagged behind: we do not yet have a good comprehension of how the structure of a graph interacts with the dimensionality of the shortest-path metric induced by it. One such example shows up in a paper [8], where a fairly simple algorithm is given for low-distortion Euclidean embeddings of unweighted trees whose shortest-path metric is doubling - however, extending the result to embed weighted trees (also with doubling shortest-path metrics) requires significantly more work. This raises the natural question: given a doubling tree metric $M=(V, d)$, is there an unweighted tree $G=\left(V^{\prime}, E^{\prime}\right)$ whose shortest-path metric is also doubling, and contains $M$ as a submetric? In fact, the situation is even more embarrassing: we do not know the answer even if we drop the requirement that $G$ be a tree, and look for any unweighted graph!

An immediate obstacle to answering these question is the observation that subdividing the edges of a weighted tree to convert it into an unweighted tree can increase the dimension unboundedly. For example, take a star $K_{1, n}$, and set the length of the $i^{t h}$ edge $\left\{v_{0}, v_{i}\right\}$ to be $2^{i}$. It is easy to check that the metric $d_{G}$ has constant doubling dimension; however, subdividing the $i^{\text {th }}$ edge into $2^{i}$ parts to make it unit-weighted creates a new graph with $n$ points at unit distance from each other, which has a doubling dimension $\log n$ that is unbounded. On the positive side, it is easy to show that this metric can be embedded into the real line with distortion 2 (e.g., the map $v_{i} \mapsto 2^{i}$ ), which we can subdivide without altering the doubling dimension. In this paper, we show that this positive result is not an aberration: any tree metric can be represented as a submetric of an unweighted tree metric which has almost the same doubling dimension. We show a similar result for arbitrary graphs as well, and show that our tradeoff between distortion and the dimension blowup is asymptotically optimal.

Formal Definitions: To define the problems we study, let us define the convex closure of a graph, which is an extension of the notion of subdividing edges. Given a graph $G=(V, E)$ with edge lengths $\ell: E \rightarrow \mathbb{R}_{\geq 0}$, assume that the names of the vertices in $V$ belong to some total order $(V, \prec)$. Let $\bar{V}_{G}$ be the uncountably infinite set of points $V \cup\{e[x] \mid e \in E, x \in(0, \ell(e))\}$ obtained by considering each edge as a continuous segment of length $\ell(e)$. Let $M_{G}=\left(V, d_{G}\right)$ be the shortestpath metric of the graph $G$ : we can define a natural metric on the set $\bar{V}_{G}$ as

$$
\begin{aligned}
\bar{d}_{G}\left(e[x], e^{\prime}[y]\right)=\min \left\{x+d\left(u, u^{\prime}\right)+y, \quad x+d\left(u, v^{\prime}\right)+\left(\ell\left(e^{\prime}\right)-y\right),\right. \\
\left.(\ell(e)-x)+d\left(u^{\prime}, v\right)+y, \quad(\ell(e)-x)+d\left(u^{\prime}, v^{\prime}\right)+\left(\ell\left(e^{\prime}\right)-y\right)\right\},
\end{aligned}
$$

if $e=\{u, v\}$ (with $u \prec v$ ) and $e^{\prime}=\left\{u^{\prime}, v^{\prime}\right\}$ (with $u^{\prime} \prec v^{\prime}$ ). We now define the convex closure of the graph $G$ to be the metric space $\operatorname{conv}(G) \doteq \bar{M}_{G}=\left(\bar{V}_{G}, \bar{d}_{G}\right)$. Note the metric obtained by subdividing edges of $G$ is a sub-metric of the convex closure of $G$, and hence it suffices to study the doubling dimension of this convex closure $\operatorname{conv}(G)$. 


\subsection{Our Results}

The example of $K_{1, n}$ with exponential edge weights shows that even if the shortest-path metric $M_{G}$ of a graph $G$ is doubling, its convex closure $\bar{M}_{G}$ may not be doubling. The goal of this paper is to show that despite this, there is a "close-by" graph $G^{\prime}$ whose convex closure $\bar{M}_{G^{\prime}}$ is indeed doubling. In particular, the main theorem is the following:

Theorem 1 (Main Theorem) Given a graph $G=(V, E)$ with specified edge-lengths, we can efficiently find a graph $G^{\prime}=\left(V, E^{\prime}\right)$ (also with non-negative edge-lengths) such that

- The distances in $G$ and $G^{\prime}$ are within a multiplicative factor of $(1+\varepsilon)$ of each other, and

- If $\operatorname{dim}\left(M_{G}\right)=k$, then $\operatorname{dim}\left(M_{G^{\prime}}\right)=O(k)$, and $\operatorname{dim}\left(\operatorname{conv}\left(G^{\prime}\right)\right)=O\left(k \log \varepsilon^{-1}\right)$.

Since Theorem 1 does not give any guarantees about the topology of the graph $G^{\prime}$, we prove an analogous result about tree metrics, with improved guarantees on the dimension:

Theorem 2 Given a tree $T=(V, E)$ with specified edge-lengths, we can efficiently find a tree $T^{\prime}=\left(V^{\prime}, E^{\prime}\right)$ with $V \subseteq V^{\prime}$ (and with non-negative edge-lengths) such that

- For $x, y \in V$, the distance between them in $T$ and $T^{\prime}$ are within a multiplicative factor of $(1+\varepsilon)$ of each other, and

- If $\operatorname{dim}\left(M_{T}\right)=k$, then $\operatorname{dim}\left(M_{T^{\prime}}\right)=O(k)$, and $\operatorname{dim}\left(\operatorname{conv}\left(T^{\prime}\right)\right)=O\left(k+\log \log \varepsilon^{-1}\right)$.

As a corollary of this result, we obtain an independent proof of the following result about embeddings of doubling tree metrics into $\ell_{p}$ spaces:

Corollary 3 ([8]) Every (weighted) doubling tree metric embeds into $\ell_{p}$ with constant distortion and constant dimension.

(Another proof of this embedding result for doubling trees appears in 20], using completely different techniques.)

In addition, we show that the tradeoff between the distortion and the dimension of the convex closure shown in Theorem 2 is asymptotically optimal:

Theorem 4 There exists a tree metric $T=(V, E)$ with $\operatorname{dim}\left(M_{T}\right)=O(1)$ such that for any tree metric $T^{\prime}=\left(V^{\prime}, E^{\prime}\right)$ with $V \subseteq V^{\prime}$, the following holds. If $d_{T}(u, v) \leq d_{T^{\prime}}(u, v) \leq(1+\varepsilon) d_{T}(u, v)$ for all $u, v \in V$, then $\operatorname{dim}\left(\operatorname{conv}\left(T^{\prime}\right)\right)$ must be $\Omega\left(\log \log \varepsilon^{-1}\right)$.

For general graphs, we show that our tradeoff is asymptotically optimal, under the restriction that the graph $G^{\prime}$ is defined on the same vertex set as $G$, i.e. we do not use any steiner points.

Theorem 5 There exists a metric $G=(V, E)$ with $\operatorname{dim}\left(M_{G}\right)=O(1)$ such that for any graph $G^{\prime}=\left(V, E^{\prime}\right)$, the following holds. If $d_{G}(u, v) \leq d_{G^{\prime}}(u, v) \leq(1+\varepsilon) d_{G}(u, v)$ for all $u, v \in V$, then $\operatorname{dim}\left(\operatorname{conv}\left(G^{\prime}\right)\right)$ must be $\Omega\left(\log \varepsilon^{-1}\right)$.

Bibliographic Note. James Lee informs us that a weaker form of Theorem 1 can be inferred from results in a paper of Semmes [24]. Indeed, the techniques of that paper imply that for every doubling metric $G$, there is a graph $G^{\prime}$ whose convex closure is also doubling; however, the resulting distortion between distances in $G$ and $G^{\prime}$ using this approach seems to depend on $\operatorname{dim}(G)$, and it is not clear how to reduce this distortion to $(1+\varepsilon)$ as in the results above. 


\subsection{Related Work}

The notion of doubling dimension was introduced by Assouad [1] and first used in algorithm design by Clarkson [5]. The properties of doubling metrics and their algorithmic applications have since been studied extensively, a few examples of which appear in [8, 17, 18, 25, 10, 2, 6, 11, 15, 16.

Somewhat similar in spirit to our work is the 0-extension problem [13, 3, 7]. Given a graph $G$, the 0-extension ( $c f$. Lipschitz Extendability [12, 22, 19]) problem deals with extending a (Euclidean) embedding of the vertices of the graph to an embedding of the convex closure of the graph, while approximately preserving the Lipschitz constant of the embedding. Our results can be interpreted as analogues to the above where the goal is to approximately preserve the doubling dimension.

A number of papers have dealt with geometric implications of topological properties of the graph inducing the metric, e.g. when the graph is planar [14, 23], outer-planar [9], series-parallel [9], or a tree [21].

\section{Preliminaries and Notation}

Given a graph $G$, the shortest path metric on it is denoted by $d_{G}$ and we shall use $B_{G}(x, r)$ to denote the "ball" $\left\{y \in V_{G}: d_{G}(x, y)<r\right\}$. We will often omit the subscript $G$ when it is obvious from context. There are several ways of defining the doubling constant $\lambda$ and the doubling dimension dim for a metric space, all of them within a constant factor of each other: here is the one that will be most useful for us.

Definition 6 (Doubling Constant and Doubling Dimension) A metric space $(X, d)$ has doubling constant $\lambda$ if for each $x \in X$ and $r \geq 0$, given the ball $B(x, 2 r)$, there is a set $S \subseteq X$ of size at most $\lambda$ such that $B(x, 2 r) \subseteq \cup_{y \in S} B(y, r)$. The doubling dimension $\operatorname{dim}((X, d))=\log _{2} \lambda$.

Fact 7 (Subset Closed) Let metric $M=(V, d)$ have doubling dimension $k$. If $X^{\prime} \subseteq X$, and $d^{\prime}=\left.d\right|_{X^{\prime} \times X^{\prime}}$, then $\left(X^{\prime}, d^{\prime}\right)$ has doubling dimension at most $k$.

Fact 8 (Small Uniform Metrics) If a metric $M=(V, d)$ has doubling dimension $k$ then there exists a point $x$ and a radius $r$ such that the ball $B(x, r)$ contains at least $2^{k}$ points with interpoint distances at least $r / 2$.

Given a metric $(X, d)$, an $r$-packing is a subset $P \subseteq X$ such that any two points in $P$ are at least distance $r$ from each other. An $r$-covering is a subset $C \subseteq X$ such that for each point $x \in X$, there is a point $c \in C$ at distance $d(x, c) \leq r$. An $r$-net is a subset $N \subseteq X$ that is both an $r$-packing and an $r$-covering.

Fact 9 ("Small" Nets) Let metric $M=(V, d)$ have doubling dimension $k$, and $N$ is an $r$-net of $M$, then for any $x \in V$ and radius $R$, the set $B(x, R) \cap N$ has size at most $(4 R / r)^{k}$.

Definition 10 (Geodesic Metrics) A metric $(X, d)$ is said to be geodesic if for every $u, v \in X$, $u \neq v$, there is a continuous map $f_{u v}:[0, d(u, v)] \rightarrow X$ such that $f_{u v}(0)=u, f_{u v}(d(u, v))=v$ and $d\left(f_{u v}(x), f_{u v}(y)\right)=|x-y|$ for any $x, y \in[0, d(u, v)]$.

Fact 11 For any graph $G=(V, E)$, the metric $\operatorname{conv}(G)$ is a geodesic metric space. 


\section{A Structure Theorem}

In this section, we show how to characterize the dimension of the convex closure of a graph $H$ in terms of some easier-to-handle parameters of the graph.

Definition 12 (Long Edges) Given a graph $H=(V, E)$, a vertex $u \in V$ and a radius $r \geq 0$, call an edge $e=\{v, w\}$ a long edge with respect to $u, r$ if one endpoint of $e$ is at distance at most $r$ from $u$, and $l(e)>r$.

Let the set of long edges with respect to $u, r$ be denoted by $L_{u}(r)$. The following structure theorem gives us a characterization of the doubling dimension of $\operatorname{conv}(H)$ in terms of the number of long edges.

Theorem 13 (Structure Theorem) There exist constants $c_{1}$ and $c_{2}$ such that the following holds. Consider any graph $H=(V, E)$, and any $k \geq \operatorname{dim}_{H}$ : if the number of long edges $\left|L_{u}(r)\right| \leq 2^{k}$ for every $u \in V$ and every $r \geq 0$, then the doubling dimension of the convex closure conv $(H)$ is at most $c_{1} k$. Moreover, if the doubling dimension of the convex closure $\operatorname{conv}(H)$ is at most $k$, then for every vertex $u \in V$ and every radius $r \geq 0$, the number of long edges $\left|L_{u}(r)\right| \leq 2^{c_{2} k}$.

Proof: Suppose the number of long edges $|L(u, r)|$ is at most $2^{k}$ for every $u, r$, then we show that for any $u \in \operatorname{conv}(H)$ and any $r>0$, the ball $B_{\text {conv }(H)}(u, 2 r)$ can be covered by at most $2^{O(k)}$ balls $B_{\text {conv }(H)}\left(y, \frac{3}{2} r\right)$. Repeating this argument three times (since $\left.\left(\frac{3}{4}\right)^{3}<\frac{1}{2}\right)$, this suffices to prove that the doubling dimension of $\operatorname{conv}(H)$ is $2^{O(k)}$.

First, consider $u \in V(H)$. From the definition of doubling dimension, there is a set $S \subset V_{H}$ of size at most $2^{2 \operatorname{dim}_{G}} \leq 2^{2 k}$ such that $V_{H} \cap B(u, 2 r) \subseteq \cup_{y \in S} B\left(y, \frac{r}{2}\right)$. Let $S^{\prime}=S \cup\{e[r] \mid e \in$ $\left.L_{u}(r)\right\} \cup\left\{e[l(e)-r] \mid e \in L_{u}(r)\right\}$. Clearly, $\left|S^{\prime}\right| \leq 2^{O(k)}$. We shall show that $B(u, 2 r)$ is contained in $\cup_{y \in S^{\prime}} B\left(y, \frac{3}{2} r\right)$.

Let $e[x] \in B(u, 2 r)$, where $e=\{v, w\}$ such that $d(u, e[x])=d(u, v)+x^{\prime}$ where $x^{\prime} \in\{x, l(e)-x\}$. Assume $v \prec w$ (the other case is similar) so that $x^{\prime}=x$. If $x \leq r$, then consider $y \in S$ such that $v \in B\left(y, \frac{r}{2}\right)$. Clearly $d(y, e[x]) \leq d(y, v)+x<\frac{3}{2} r$. Hence $e[x] \in B\left(y, \frac{3}{2} r\right)$. On the other hand, if $x>r$ and $e[x] \in B(u, 2 r)$, then $d(u, v)=d(u, e[x])-x \leq r$ so that the edge $e$ is long with respect to $(u, r)$. Thus $e[r] \in S^{\prime}$ and since $0 \leq x \leq 2 r$, we conclude that $e[x] \in B(e[r], r)$. Thus for any $u \in V_{H}, B(u, 2 r) \subseteq \cup_{y \in S^{\prime}} B\left(y, \frac{3}{2} r\right)$.

Finally, we have to consider balls around vertices in $\operatorname{conv}(H) \backslash V(H)$ : note that for $e=\{u, v\}$, $B(e[x], 2 r) \subseteq B(u, 2 r) \cup B(v, 2 r) \cup\{e[z] \mid \max (0, x-2 r) \leq z \leq \min (l(e), x+2 r)\}$. By the argument above, the first two can be covered by a $2^{O(k)}$ balls of radius $r$ each. The subset of $e$ in $B(e[x], 2 r)$ is one dimensional and thus can be covered by two balls of radius $r$ each. This completes the argument showing that if the number of long edges is small, the convex completion has a small doubling dimension.

For the converse, we shall show that $\operatorname{dim}(\operatorname{conv}(H)) \geq \Omega\left(\log \max _{u, r} L_{u}(r)\right)$. Indeed consider the set of points $W=\left\{e\left[\frac{r}{2}\right] \mid r \in L_{u}(r)\right\}$. It is easy to see that $W \subseteq B(u, 2 r)$ but the balls $\left\{B\left(w, \frac{r}{2}\right) \mid w \in W\right\}$ are all disjoint. Thus $\operatorname{dim}(\operatorname{conv}(H)) \geq \frac{1}{2} \log |W|=\frac{1}{2} \log \left|L_{u}(r)\right|$, whence the claim follows.

The following simple result follows immediately.

Corollary 14 For any $n$ point metric $(X, d)$, there exists a geodesic metric $\left(X^{\prime}, d^{\prime}\right)$ that contains an isometric copy of $(X, d)$ and has doubling dimension at most $O(\log n)$. 
The example of $K_{1, n}$ with exponential edge weights shows that this bound is tight, even when $(X, d)$ itself has constant doubling dimension. In the following sections, we show that when $(X, d)$ indeed has small doubling dimension, the $O(\log n)$ bound above can be improved considerably if one allows a small distortion.

\section{Convex Completions for Graphs: Proof of Main Theorem}

In this section, we show how to take a graph $G=(V, E)$ and obtain a graph $G^{\prime}=\left(V, E^{\prime}\right)$ on the same vertex set, which has (almost) the same distances as in $G$, but whose doubling dimension does not change by much under taking the convex closure. In particular, we use a bounded-degree spanner construction due to Chan et al. 4]: they give an algorithm that given a metric $(V, d)$ with dimension $\operatorname{dim}=\operatorname{dim}(G)$ and a parameter $\varepsilon<1 / 4$, outputs a spanner $G^{\prime}=\left(V, E^{\prime}\right)$ such that $d(x, y) \leq d_{G^{\prime}}(x, y) \leq(1+\varepsilon) d(x, y)$ for all pairs $x, y \in V$, and moreover the degree of each vertex $x \in V$ is bounded by $\varepsilon^{-O\left(\operatorname{dim}_{G}\right)}$. We show that the convex closure of this spanner has doubling dimension of $O\left(\operatorname{dim}_{G} \log \varepsilon^{-1}\right)$.

\subsection{The Spanner Construction}

We start with a graph $G$ and carry out a series of transformations to obtain graph $G^{\prime}$. Let $\varepsilon<\frac{1}{4}$ be given and let $\tau=6+\left\lceil\log \left(\frac{1}{\varepsilon}\right)\right\rceil$. Without loss of generality, the smallest pairwise distance in $G$ is at least $2^{\tau}$. We start with some more definitions.

Definition 15 (Hierarchical Tree) A hierarchical tree for a set $V$ is a pair $(\mathbb{T}, \phi)$, where $\mathbb{T}$ is a rooted tree, and $\phi$ is a labeling function $\phi: \mathbb{T} \rightarrow V$ that labels each node of $\mathbb{T}$ with an element in $V$, such that the following conditions hold.

1. Every leaf is at the same depth from the root.

2. The function $\phi$ restricted to the leaves of $\mathbb{T}$ is a bijection into $V$.

3. If $u$ is an internal node of $\mathbb{T}$, then there exists a child $v$ of $u$ such that $\phi(v)=\phi(u)$. This implies that the nodes mapped by $\phi$ to any $x \in V$ form a connected subtree of $\mathbb{T}$.

Definition 16 (Net-Tree) A net tree for a metric $(V, d)$ is a hierarchical tree $(\mathbb{T}, \phi)$ for the set $V$ such that the following conditions hold.

1. Let $N_{i}$ be the set of nodes of $\mathbb{T}$ that have height $i$. (The leaves have height 0 .) Let $r_{0}=1$, and $r_{i+1}=2 r_{i}$, for $i \geq 0$. (Hence, $r_{i}=2^{i}$.) Then, for $i \geq 0, \phi\left(N_{i+1}\right)$ is an $r_{i+1}$-net for $\phi\left(N_{i}\right)$.

2. Let node $u \in N_{i}$, and its parent node be $p_{u}$. Then, $d\left(\phi(u), \phi\left(p_{u}\right)\right) \leq r_{i+1}$.

It is easy to see that net-trees exist for all metrics, and Har-Peled and Mendel show how to construct a net-tree efficiently [10].

To construct their bounded-degree spanner, Chan et al. [4] define the following: suppose we are given a graph $G=(V, E)$, whose shortest-path metric $\left(V, d_{G}\right)$ has doubling dimension $\operatorname{dim}_{G}$. Let $\varepsilon>0$ and $(\mathbb{T}, \phi)$ be any net tree for $M$. For each $i>0$, let

$$
E_{i}:=\left\{\{u, v\} \mid u, v \in \phi\left(N_{i}\right), d_{G}(u, v) \leq\left(4+\frac{32}{\varepsilon}\right) \cdot r_{i}\right\} \backslash \bigcup_{j \leq i-1} E_{j},
$$


where $E_{0}$ is the empty set. (Here the parameters $N_{i}, r_{i}$ are as in Definition [16]) Letting $C_{\varepsilon}$ denote $\left(4+\frac{32}{\varepsilon}\right)$, we note that all edges in $E_{i}$ have length in $\left(C_{\varepsilon} r_{i-1}, C_{\varepsilon} r_{i}\right]$.

While the graph $\widehat{G}=\left(V, \widehat{E}=\cup_{i} E_{i}\right)$ is a $(1+\varepsilon)$-spanner for the original metric with few edges, obtaining a bounded-degree spanner requires some modifications to the basic construction. First, the edges in $\widehat{E}$ are directed (merely for the purposes of the algorithm, and the proof). For each $v \in V$, define $i^{*}(v):=\max \left\{i \mid v \in \phi\left(N_{i}\right)\right\}$. For each edge $(u, v) \in \widehat{E}$, we direct the edge from $u$ to $v$ if $i^{*}(u)<i^{*}(v)$. If $i^{*}(u)=i^{*}(v)$, the edge can be directed arbitrarily. Chan et al. show that each vertex $x \in V$ has out-degree bounded by $\beta=\varepsilon^{-O\left(\operatorname{dim}_{G}\right)}$. Then, the following steps are performed:

- Consider any vertex $x$, and all the edges that are directed into $x$. These edges come from various sets $E_{i}$ : let us denote by $F_{i}=F_{i}(x)$ the subset of edges directed into $x$ that belong to $E_{i}$.

- Suppose the non-empty subsets are $F_{i_{1}}, F_{i_{2}}, \ldots, F_{i_{t}}$, where $i_{j}<i_{j+1}$. We do nothing to the first $7 \log \varepsilon^{-1}$ of these edge sets; these contribute $\varepsilon^{-O\left(\operatorname{dim}_{G}\right)}$ to the final degree of $x$.

- Consider a value of $j>7 \log \varepsilon^{-1}$ : from the set $F_{i_{\left(j-7 \log \varepsilon^{-1}\right)}}$ of edges directed into $x$, we choose an arbitrary one $\{u, x\}$. We replace edges of the form $\{y, x\} \in F_{i_{j}}$ by edges $\{y, u\}$-and refer to these (at most $\varepsilon^{-O\left(\operatorname{dim}_{G}\right)}$ ) edges as edges donated from $x$ to $u$.

Note that the length of the edge $\{u, x\}$ is at most $C_{\varepsilon} 2^{i}\left(j-7 \log \varepsilon^{-1}\right) \leq C_{\varepsilon} \varepsilon^{7} 2^{i}$, whereas the length of any edge in $\{y, x\} \in F_{i_{j}}$ is at least $C_{\varepsilon} 2^{i-1}$; hence $d_{G}(u, x) \leq\left(\varepsilon^{7} / 2\right) d_{G}(x, y) \leq \varepsilon^{6} d_{G}(x, y)$, since $\varepsilon \leq 1 / 4$. By the triangle inequality, $d_{G}(u, y) \in\left(1 \pm \varepsilon^{6}\right) d_{G}(x, y)$.

Additionally, note that if $x$ donates a long edge $(x, y) \in F_{i_{j}}$ to $u$, then $(u, x) \in F_{i_{j-7 \log \varepsilon^{-1}}}$ so that $d_{G}(x, u)$ is at least $C_{\varepsilon} 2^{\left(i_{\left.j-7 \log \varepsilon^{-1}\right)-1}\right.}$.

Theorem 17 ([4]) The spanner thus constructed has degree $\varepsilon^{-O\left(\operatorname{dim}_{G}\right)}$ and stretch $(1+\varepsilon)$.

From the construction of the bounded-degree spanner, note that each vertex $u \in V$ has the following edges incident to it:

- Type-A edges. These correspond to the $\varepsilon^{-O\left(\operatorname{dim}_{G}\right)}$ edges that were directed away from $u$.

- Type-B edges. These correspond to the edges directed into $u$ that belong to the smallest $7 \log \varepsilon^{-1}$ levels; this gives another $\left(\varepsilon^{-O\left(\operatorname{dim}_{G}\right)}\right)$ edges in total.

- Type-C edges. For each edge $e=\{u, x\}$ of type-A incident to $u$, there are at most $\left(\varepsilon^{-O\left(\operatorname{dim}_{G}\right)}\right)$ other edges incident to $u$ that are not counted above. Each such edge $e^{\prime}=\{y, u\}$ corresponds to some edge of the form $\{y, x\} \in E_{i}$ (for some $i$ such that $x, y \in \phi\left(N_{i}\right)$ ), such that the edge was "donated" from $x$ to $u$ to maintain $x$ 's degree bound.

\subsection{Bounding the Dimension of the Convex Closure}

Simply by the distortion bound, it follows that the doubling dimension of the bounded-degree spanner $G^{\prime}$ is close to $\operatorname{dim}_{G}$. Of course, the bounded-degree does not imply that $\operatorname{conv}\left(G^{\prime}\right)$ has low doubling dimension: in this section, we use the Structure Theorem 13 to show this fact, and hence prove Theorem 1.

Lemma 18 Given the graph $G^{\prime}$ defined as above, fix any vertex $v$ and radius $R$, and $\varepsilon<\frac{1}{4}$. Then the number of long edges $\left|L_{v}(R)\right|$ with respect to $v, R$ is at most $O\left(\varepsilon^{-O\left(\operatorname{dim}_{G}\right)}\right)$. 
Proof: Recall that $L_{v}(R)$ is the set of edges that have one endpoint within the ball $B(v, R)$, and have length at least $R$. Define $\ell \in \mathbb{Z}_{\geq 0}$ such that $R \in\left(C_{\varepsilon} 2^{\ell-1}, C_{\varepsilon} 2^{\ell}\right]$.

By the spanner construction, any type-A or type-B edge that is long must belong to $\cup_{i \geq \ell} E_{i}$, and hence must have both endpoints in $\phi\left(N_{\ell}\right)$. Moreover, one endpoint of each such a long edge must lie in the ball $B(v, R) \subseteq B\left(v, C_{\varepsilon} 2^{\ell}\right)$; since the points in $\phi\left(N_{\ell}\right)$ are at distance at least $2^{\ell}$ from each other, there can be at most $\left(C_{\varepsilon}\right)^{O\left(\operatorname{dim}_{G}\right)}$ many such endpoints within the ball. Moreover, each one of these endpoints has at most $\varepsilon^{-O\left(\operatorname{dim}_{G}\right)}$ type-A or type-B edges; multiplying them together, using the fact that $C_{\varepsilon}=O\left(\varepsilon^{-1}\right)$, and simplifying gives an upper bound of $\varepsilon^{-O\left(\operatorname{dim}_{G}\right)}$ on the number of type-A and type-B edges in $L_{v}(R)$.

Let us now consider the edges in $L_{v}(R)$ that are of type-C with respect to their endpoint within $B(v, R)$. Recall that each type-C edge $\{u, y\}$ can be associated with some edge $\{x, y\} \in \widehat{E}$ (of almost the same length - up to a factor of $\left.\left(1 \pm \varepsilon^{6}\right)\right)$ such that $x$ donates the edge to $u$. Let us fix one such long edge $e=\{u, y\}$ associated with $\{x, y\} \in E_{i}$-hence the distance $d_{G}(x, y) \in\left(C_{\varepsilon} 2^{i-1}, C_{\varepsilon} 2^{i}\right]$, and also $x, y \in \phi\left(N_{i}\right)$. By the construction of the type-C edges, the distance $d_{G}(u, x) \leq \varepsilon^{6} \cdot d_{G}(x, y)$, and hence $x$ lies in the ball $B\left(v, R+\varepsilon^{6} C_{\varepsilon} 2^{i}\right)$.

Given any fixed level $i \geq \ell-1$, the number of donor vertices is bounded by the number of points in $B\left(v, C_{\varepsilon}\left(2^{\ell}+\varepsilon^{6} 2^{i}\right)\right)$ that are at least $2^{i}$ distance apart from each other, which can be loosely bounded by $\varepsilon^{-O\left(\operatorname{dim}_{G}\right)}$. Each such donor vertex could donate $\varepsilon^{-O\left(\operatorname{dim}_{G}\right)}$ edges, which would give us a total of $\varepsilon^{-O\left(\operatorname{dim}_{G}\right)}$ edges for the level $i$. Summing this over all levels would give us too many edges, so we use this bound only for levels $i$ such that $\ell-1 \leq i \leq \ell+O\left(\log \varepsilon^{-1}\right)$.

Consider any level $i>\ell+6 \log \varepsilon^{-1}$ : any donor vertex for such a level must lie in the ball $B\left(v, C_{\varepsilon}\left(2^{\ell}+\right.\right.$ $\left.\left.\varepsilon^{6} 2^{i}\right)\right) \subseteq B\left(v, C_{\varepsilon} \varepsilon^{6} 2^{i+1}\right) \subseteq B\left(v, C_{\varepsilon} \varepsilon^{5} 2^{i}\right)$. A little algebra shows that

$$
\varepsilon^{5} C_{\varepsilon}=\varepsilon^{5}\left(4+\frac{32}{\varepsilon}\right) \leq \varepsilon^{5} \frac{33}{\varepsilon} \leq \varepsilon^{4} \cdot 33 \leq \varepsilon,
$$

and thus the donor vertex must be at distance at most $\varepsilon 2^{i}$ from $v$. However, since the donor vertex must belong to $\phi\left(N_{i}\right)$, it must be at distance at least $2^{i}$ from any other donor vertices. Now, if there were two donor vertices at distance $\varepsilon 2^{i}$ from $v$, they would be at distance $2 \varepsilon 2^{i}<2^{i}$ from each other - this implies that there can be at most one donor vertex for such a "high" level.

Finally, it remains to show that the total number of long edges donated by this donor vertex $x$ to vertices in $B(v, R)$ is small. Let $i_{1}, i_{2}, \ldots, i_{t}, i_{j}<i_{j+1}$ be the levels for which $x$ donates a long edge to vertices in $B(v, R)$; we shall show that $t$ is at most $O\left(\log \varepsilon^{-1}\right)$. Since the first edge is long, $R \leq C_{\varepsilon} 2^{i_{1}+1}$. Moreover, since $x$ donates this edge to $u$, we conclude that $d_{G}\left(x, u_{1}\right) \leq \varepsilon^{6} C_{\varepsilon} 2^{i_{1}}$, so that $d_{G}(v, x) \leq R+\varepsilon^{6} C_{\varepsilon} 2^{i_{1}} \leq\left(2+\varepsilon^{6}\right) C_{\varepsilon} 2^{i_{1}}$. Suppose that $t>7 \log \varepsilon^{-1}+3$. Then an edge in $F_{i_{t}}$ is donated from $x$ to $u_{t}$, and we have that $d_{G}\left(x, u_{t}\right) \geq C_{\varepsilon} 2^{i_{4}-1}$. On the other hand, since $u_{t} \in B(v, R)$, by triangle inequality, $d_{G}\left(x, u_{t}\right) \leq d_{G}(x, v)+d_{G}\left(v, u_{t}\right) \leq\left(3+\varepsilon^{6}\right) C_{\varepsilon} 2^{i_{1}}$. Since $i_{4} \geq i_{1}+3$, this gives us the desired contradiction. Thus $t \leq O\left(\log \varepsilon^{-1}\right)$. Since there are at most $\varepsilon^{-O\left(\operatorname{dim}_{G}\right)}$ edges donated to $B(v, R)$ from each of these levels, the claim follows.

Using Lemma 18 along with the Structure Theorem 13 implies that the dimension of $\operatorname{conv}\left(G^{\prime}\right)$ is bounded by $O\left(\operatorname{dim}_{G} \log \varepsilon^{-1}\right)$, which proves Theorem 1 , 


\section{Convex Completions for Trees}

The construction of the previous section showed that given any graph $G$, we could construct a new graph $G^{\prime}$ such that distances in $G$ and $G^{\prime}$ are within $(1+\varepsilon)$ of each other, and $\operatorname{conv}\left(G^{\prime}\right)$ has low doubling dimension. However, since the construction starts with the shortest-path metric $d_{G}$ and completely ignores the topological structure of $G$ itself, it is not suited to proving Theorem 2 which seeks to start with a tree and end with another tree. In this section, we show a different approach that allows us to monitor the graph structure more closely.

\subsection{The Construction for Trees}

We give a procedure that takes a general graph $G$ and outputs a graph $G^{\prime}$ (since the construction itself does not depend on $G$ being a tree); we then show some properties that hold when $G$ is a tree. The procedure takes a graph $G=(V, E)$, and constructs a new graph $G^{\prime}=\left(V^{\prime}, E^{\prime}\right)$ with $V \subseteq V^{\prime}$ (by way of an intermediate graph $\widehat{G}$ ) as follows. Define an exponential tail with $k$ edges as a path $P=\left\langle v_{0}, v_{1}, v_{2}, \ldots, v_{k}\right\rangle$, where the length of the edge $\left\{v_{i-1}, v_{i}\right\}$ is $2^{i}$. Without loss of generality, the smallest edge length in $G$ is at least $2^{\tau}$, where $\tau=6+\left\lceil\log \left(\frac{1}{\varepsilon}\right)\right\rceil$.

We construct the graph $G^{\prime}$ in the following way:

- As in Section 4.1, we consider a net-tree $(\mathbb{T}, \phi)$ for the graph $G$. If $N_{i}$ is the set of nodes in $T$ at height $i$, then for $u \in V$ define $i^{*}(u)$ to be the largest $i$ such that $u \in \phi\left(N_{i}\right)$. Attach to each $u \in V$ an exponential tail with $i^{*}(u)$ edges; refer to the $j^{\text {th }}$ vertex on this path as $u_{[j]}$, with $u_{[0]}=u$. Let $\widehat{G}$ be this intermediate graph consisting of $G$ along with the tails.

- Consider an edge $e=\{u, v\} \in E(G)$, and suppose its length lies in the interval $\left(C_{\varepsilon} 2^{i-1}, C_{\varepsilon} 2^{i}\right]$. Some leaf of $T$ must be mapped by $\phi$ to $u \in V$ : let the level-(i) ancestor of that node be mapped by $\phi$ to $\widehat{u}$; similarly, define $\widehat{v}$ be defined for $v$. We now make an edge $\left\{\widehat{u}_{[i]}, \widehat{v}_{[i]}\right\}$ of length $\ell_{e}$ in the graph $G^{\prime}$.

Note that if we start off with a tree $T$, the above procedure adds exponential tails to $T$ to get the intermediate graph $\widehat{T}$, and then "moves the edges up the tails" to get the final graph $T^{\prime}$.

Proposition 19 (Distance Preservation) Let $\varepsilon<1 / 4$. If the input graph is a tree $T=(V, E)$, then the above procedure results in a connected tree $T^{\prime}=\left(V^{\prime}, E^{\prime}\right)$ such that for any $x, y \in V$,

$$
(1+\varepsilon)^{-1} d_{T}(x, y) \leq d_{T^{\prime}}(x, y) \leq(1+\varepsilon) d_{T}(x, y) .
$$

Proof: Let us consider performing the above-mentioned transformation for edges in increasing order of edge-length. Given $j \in \mathbb{Z}_{\geq 0}$, let $T_{j}$ be the forest formed by deleting all edges of length more than $C_{\varepsilon} 2^{j}$ from $T$; also, let $T_{j}^{\prime}$ be the forest formed by deleting the corresponding edges in $T^{\prime}$. We will prove by induction on $j$ that for all $x, y$ that lie in some connected component in $T_{j}$, their distance in $T_{j}^{\prime}$ will satisfy the desired stretch bound. The base case is trivial, since all components of $T_{0}$ have single nodes in them.

To prove the claim for $j$, we inductively assume it for $j-1$. Now consider taking some edge $e=\{u, v\}$ of length $\ell_{e} \in\left(C_{\varepsilon} 2^{j-1}, C_{\varepsilon} 2^{j}\right]$. In this case we find some nodes $\widehat{u}$ and $\widehat{v}$, and add an edge of length $\ell_{e}$ between $\widehat{u}_{[j]}$ and $\widehat{v}_{[j]}$. By the properties of the net-tree, the distance $d_{T}(u, \widehat{u}) \leq 2^{j+1}-2$. Since $T_{j}$ already contains all edges of length at most $C_{\varepsilon} 2^{j-1}$, and $C_{\varepsilon} \geq 4$, the net point $\widehat{u}$ lies in the 
same component as $u$ in $T_{j}$. By the induction hypothesis, $d_{T^{\prime}}(u, \widehat{u}) \leq(1+\varepsilon) 2^{j+1}$; note that this implicitly proves that $u$ and $\widehat{u}$ are in the same component in $T_{j}^{\prime}$. A similar claim holds for $d_{T}(v, \widehat{v})$. Hence the distance in $T_{j+1}^{\prime}$ between $u$ and $v$ is at most

$$
\begin{aligned}
& d_{T_{j}^{\prime}}(u, \widehat{u})+d_{T_{j}^{\prime}}\left(\widehat{u}, \widehat{u}_{[j]}\right)+\ell_{e}+d_{T_{j}^{\prime}}\left(\widehat{v}_{[j]}, \widehat{v}\right)+d_{T_{j}^{\prime}}(\widehat{v}, v) \\
& =2 \times(1+\varepsilon) 2^{j+1}+2 \times 2^{j+1}+\ell_{e} \\
& \leq \ell_{e}\left(\frac{8(1+\varepsilon)+8}{C_{\varepsilon}}+1\right) \leq(1+\varepsilon) \ell_{e},
\end{aligned}
$$

where we used the fact that $C_{\varepsilon}=\left(4+\frac{32}{\varepsilon}\right)$ and $\varepsilon<1 / 4$. Since each of the edges of $T$ are not stretched by more than $(1+\varepsilon)$, this implies that the stretch for all pairs is bounded by the same value.

We also need to show that the distances are not shrunk too much in $T^{\prime}$ : to show this, we go via $\widehat{T}$. (Recall that $\widehat{T}$ was the original tree $T$ along with the exponential tails.) First note that for any $u, v \in V, d_{T}(u, v)=d_{\widehat{T}}(u, v)$. We show that distance do not shrink in going from $\widehat{T}$ to $T^{\prime}$. It suffices to show this for the edges of $T^{\prime}$. For an edge $e^{\prime}=\left(\widehat{u}_{[j]}, \widehat{v}_{[j]}\right)$ that has length $\ell_{e} \geq C_{\varepsilon} 2^{j-1}$, we note that their distance in $\widehat{T}$

$$
d_{\widehat{T}}\left(\widehat{u}_{[j]}, \widehat{v}_{[j]}\right) \leq d_{\widehat{T}}\left(\widehat{u}_{[j]}, \widehat{u}\right)+d_{\widehat{T}}(\widehat{u}, u)+\ell_{e}+d_{\widehat{T}}(v, \widehat{v})+d_{\widehat{T}}\left(\widehat{v}, \widehat{v}_{[j]}\right) \leq 4\left(2^{j+1}-2\right)+\ell_{e}
$$

Since $C_{\varepsilon}>32 / \varepsilon$, this is at most $(1+\varepsilon) \ell_{e}$. Thus the contraction going from $\widehat{T}$ to $T^{\prime}$ is at most $(1+\varepsilon)$.

Finally, we note that we have shown that $T^{\prime}$ is connected, and the number of edges in $T^{\prime}$ is equal to the number of edges in $\widehat{T}$, which is a tree. Thus $T^{\prime}$ is a tree as well.

\subsection{Bounding the Dimension of the Convex Closure: The Tree Case}

Finally, to show that the doubling dimension of conv $\left(T^{\prime}\right)$ is small, we will again invoke Theorem 13 , However, since we have added additional vertices in going from $T$ to $T^{\prime}$, we first show that $\operatorname{dim}\left(T^{\prime}\right)$ is $O(\operatorname{dim}(T))$. Since we have already shown that distances are preserved in going from $\widehat{T}$ to $T^{\prime}$, it suffices to bound the doubling dimension of $\widehat{T}$.

Lemma 20 The doubling dimension of $\widehat{T}$ is at most $O(\operatorname{dim}(T))$.

Proof: Let $u_{[i]} \in V(\widehat{T})$ and $R \geq 0$ with $R \in\left(2^{j-1}, 2^{j}\right]$. We wish to show that $B\left(u_{[i]}, 2 R\right)$ can be covered by a small number of balls of radius $R$. From the definition of doubling dimension, it follows that there is a set $Y$ with $|Y| \leq 2^{2 \operatorname{dim}(T)}$ such that $B_{T}(u, 2 R) \subseteq \cup_{y \in Y} B_{T}(u, R / 2)$. Note that for any $v \notin \phi\left(N_{j-2}\right)$, the tail attached to $v$ has length at most $R / 2$. Let $Z=B(u, 2 R) \cap \phi\left(N_{j-2}\right)$; clearly $|Z| \leq 2^{O(\operatorname{dim}(T))}$. Finally, let $Z^{\prime}=\left\{v_{[j-1]}: v \in Z\right\}$ and $Z^{\prime \prime}=\left\{v_{[j]}: v \in Z\right\}$. It is easy to

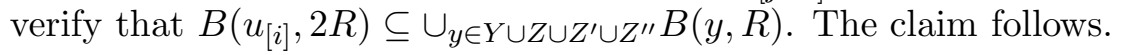

Finally, we show the following bound on the number of long edges in $T^{\prime}$.

Lemma 21 (Few Long Edges) For any vertex $v \in T^{\prime}$ and every radius $R$, the number of long edges in $T^{\prime}$ is bounded by $2^{O(\operatorname{dim})} \log \varepsilon^{-1}$. 
Proof: First consider some $v \in V$, and $R \geq 0$, and define $\ell \in \mathbb{Z}_{\geq 0}$ such that $R \in\left(C_{\varepsilon} 2^{\ell-1}, C_{\varepsilon} 2^{\ell}\right]$. Every long edge incident on $B(v, R)$ must have length at least $R$. Further, edges longer than $2 C_{\varepsilon} R$ are incident on a tail node further than $R$ from its root, and hence such an edge cannot be incident on $B(v, R)$. For each of the length scales $\left(C_{\varepsilon} 2^{\ell+j-1}, C_{\varepsilon} 2^{\ell+j}\right): 0 \leq j \leq \log C_{\varepsilon}$, we will bound the number of long edges in that length scale. Fix one such scale, and let $L(v, R, j)=\left\{\left(u_{i}, w_{i}\right): 1 \leq i \leq\right.$ $|L(v, R, j)|\}$ be the set of long edges of length in $\left(C_{\varepsilon} 2^{\ell+j-1}, C_{\varepsilon} 2^{\ell+j}\right)$, such that $d\left(v, u_{i}\right) \leq R$. Since each long edge has length more than $R$, there is a path from $v$ to $u_{i}$ that does not use any of the long edges. Consider the set of nodes $W=\left\{w_{i}: 1 \leq i \leq|L(v, R, j)|\right\}$. Clearly, for any $w, w^{\prime} \in W$, $d\left(w, w^{\prime}\right)$ is at most $2 R+2 C_{\varepsilon} 2^{\ell+j} \leq 4 C_{\varepsilon} 2^{\ell+j}$. Moreover, since $T$ is a tree, the symmetric difference of the $v-w$ and $v-w^{\prime}$ paths gives the shortest path from $w^{\prime}$ to $w$. Since the long edges incident on $w$ and $w^{\prime}$ are in this symmetric difference, we conclude that $d\left(w, w^{\prime}\right) \geq 2 C_{\varepsilon} 2^{\ell+j-1}$. Thus from the bound on doubling dimension, we conclude that $|W| \leq 2^{O(\operatorname{dim})}$. Adding the contribution of the $O\left(\log \varepsilon^{-1}\right)$ distance scales, we get the desired bound.

We now extend the argument to a vertex $v_{[i]}$ on an exponential tail hanging off $v$. If $i \geq j$, then $B\left(v_{[i]}, R\right)=\left\{v_{[i]}\right\}$. All edges incident on $v$ have, up to a factor of two, the same length, and thus their endpoints form a near uniform submetric. Thus we can bound the degree of $v_{[i]}$ by $2^{O(\operatorname{dim})}$ and the claim follows. On the other hand, when $i<j, B\left(v_{[i]}, R\right) \subseteq B(v, 2 R)$ and an argument analogous to the one for the case $v \in V$ above suffices.

Theorem 2 follows.

\section{Lower Bounds}

In this section, we show that the tradeoff between distortion and dimension blowup is asymptotically optimal. Consider the graph $K_{1, n}$ with $v_{0}$ as the center node and $\left\{v_{1}, \ldots, v_{n}\right\}$ as the set of leaves. Set the length of the edge $\left\{v_{0}, v_{i}\right\}$ to $2^{i}$ and let $d$ be the resulting metric on the vertices $V$ of $K_{1, n}$. It is easy to check that this metric has constant doubling dimension. We next show that the doubling dimension of any geodesic metric $\left(X, d^{\prime}\right)$ containing a $(1+\varepsilon)$-distortion copy of $(V, d)$ is $\Omega\left(\log \log \varepsilon^{-1}\right)$.

Lemma 22 Let $\left(X, d^{\prime}\right)$ be any geodesic metric such that $V \subseteq X$ and $d\left(v_{i}, v_{j}\right) \leq d^{\prime}\left(v_{i}, v_{j}\right) \leq(1+$ $\varepsilon) d\left(v_{i}, v_{j}\right)$ for all $v_{i}, v_{j} \in V$. Then $\operatorname{dim}\left(X, d^{\prime}\right)$ is $\Omega\left(\log \log \varepsilon^{-1}\right)$.

Proof: Denote by $u w[x]$ the point on the shortest $u$-w path in $X$ that is at distance $x$ from $u$ (if there is more than one shortest path, pick one arbitrarily). We shall argue that the points $v_{0} v_{i}[1]$ for $i=\left\{1, \ldots, \log (2 \varepsilon)^{-1}\right\}$ form a large near-uniform submetric in $X$. Indeed $d^{\prime}\left(v_{0} v_{i}[1], v_{0} v_{j}[1]\right) \leq$ $d^{\prime}\left(v_{0} v_{i}[1], v_{0}\right)+d^{\prime}\left(v_{0}, v_{0} v_{j}[1]\right)=2$. On the other hand, by triangle inequality,

$$
\begin{aligned}
d^{\prime}\left(v_{0} v_{i}[1], v_{0} v_{j}[1]\right) & \geq d^{\prime}\left(v_{i}, v_{j}\right)-d^{\prime}\left(v_{0} v_{i}[1], v_{i}\right)-d^{\prime}\left(v_{0} v_{j}[1], v_{j}\right) \\
& =d^{\prime}\left(v_{i}, v_{j}\right)-\left(d^{\prime}\left(v_{0}, v_{i}\right)-1\right)-\left(d^{\prime}\left(v_{0}, v_{j}\right)-1\right) \\
& \geq 2+d\left(v_{i}, v_{j}\right)-(1+\varepsilon)\left(d\left(v_{0}, v_{i}\right)+d\left(v_{0}, v_{j}\right)\right) \\
& =2-\varepsilon\left(2^{i}+2^{j}\right)
\end{aligned}
$$

where we have used the bound on the distortion and the distance definitions in $d$ in the last two steps. Since $i, j \leq \log (2 \varepsilon)^{-1}$, we conclude that $d^{\prime}\left(v_{0} v_{i}[1], v_{0} v_{j}[1]\right) \geq 1$. Thus we have $\log (2 \varepsilon)^{-1}$ 
points in $X$ that lie within $B\left(v_{0}, 2\right)$ no two of which can be covered by a single ball of radius $\frac{1}{2}$. Thus the doubling dimension of $X$ is $\Omega\left(\log \log \varepsilon^{-1}\right)$.

Theorem 4 follows.

For general metrics, we show a stronger lower bound, under a stronger constraint on $X$. Let $V=\{0,1\}^{p}$ with $d(x, y)=2^{p-l c p(x, y)}$, where $l c p(x, y)$ denotes the length of the longest common prefix of strings $x$ and $y$. Once again, one can easily check that $(V, d)$ has constant doubling dimension. We show that any graph $H=(V, E)$ on $V$ approximating $d$ within distortion $(1+\varepsilon)$ must satisfy $\operatorname{dim}(\operatorname{conv}(H)) \in \Omega\left(\log \varepsilon^{-1}\right)$.

Lemma 23 Let $H=(V, E)$ be any graph such that the shortest path metric $d^{\prime}$ satisfies $d(x, y) \leq$ $d^{\prime}(x, y) \leq(1+\varepsilon) d(x, y)$ for all $x, y \in V$. Then $\operatorname{dim}(\operatorname{conv}(H))$ is $\Omega\left(\log \varepsilon^{-1}\right)$.

Proof: For $p=\log (2 \varepsilon)^{-1}$, we first show that $H$ must have all edges connecting $V_{0}=\{0 x: x \in$ $\left.\{0,1\}^{p-1}\right\}$ and $V_{1}=\left\{1 x: x \in\{0,1\}^{p-1}\right\}$. Indeed, suppose that edge $(0 x, 1 y) \notin H$. Then the shortest path in $H$ between $0 x$ and $1 y$ must be of length at least $2^{p}+1$. This however violates the distortion constraint. Now consider the set of points $A=\left\{e\left[2^{p-1}\right]: e=\left(0 x, 1 y, x, y \in\{0,1\}^{p-1}\right\}\right.$. Clearly for any $a, b \in A, d(a, b) \leq 3 \cdot 2^{p-1}$ and $d(a, b) \geq 2 \cdot 2^{p-1}$. The claimed bound on the doubling dimension follows.

Theorem 5 follows.

\section{Acknowledgments}

We thank James Lee for pointing out that a weaker version of Theorem 1 could be inferred from Semmes' results. We also thank Robi Krauthgamer and Ravishankar Krishnaswamy for discussions.

\section{References}

[1] P. Assouad. Plongements lipschitziens dans $\mathbf{R}^{n}$. Bull. Soc. Math. France, 111(4):429-448, 1983.

[2] A. Beygelzimer, S. Kakade, and J. Langford. Cover trees for nearest neighbor. In The 23rd International Conference on Machine Learning (ICML), 2006.

[3] G. Călinescu, H. Karloff, and Y. Rabani. Approximation algorithms for the 0-extension problem. In Proceedings of the twelfth annual ACM-SIAM symposium on Discrete algorithms, pages 8-16. ACM Press, 2001.

[4] H. T.-H. Chan, A. Gupta, B. M. Maggs, and S. Zhou. On hierarchical routing in DOubling metrics. In Proceedings of the 16th ACM-SIAM Symposium on Discrete Algorithms (SODA), pages $762-771,2005$.

[5] K. L. Clarkson. Nearest neighbor queries in metric spaces. Discrete Comput. Geom., 22(1):6393, 1999.

[6] R. Cole and L.-A. Gottlieb. Searching dynamic point sets in spaces with bounded doubling dimension. In The thirty-eighth annual ACM symposium on Theory of computing (STOC), 2006 . 
[7] J. Fakcharoenphol, C. Harrelson, S. Rao, and K. Talwar. An improved approximation algorithm for the 0-extension problem. In Proceedings of the fourteenth annual ACM-SIAM symposium on Discrete algorithms, pages 257-265. Society for Industrial and Applied Mathematics, 2003.

[8] A. Gupta, R. Krauthgamer, and J. R. Lee. Bounded geometries, fractals, and low-distortion embeddings. In Proceedings of the 44th Symposium on the Foundations of Computer Science (FOCS), pages 534-543, 2003.

[9] A. Gupta, I. Newman, Y. Rabinovich, and A. Sinclair. Cuts, trees and $\ell_{1}$-embeddings of graphs. Combinatorica, 24(2):233-269, 2004. (Preliminary version in 40th FOCS, 1999.).

[10] S. Har-Peled and M. Mendel. Fast constructions of nets in low dimensional metrics, and their applications. In Proceedings of the twenty-first annual symposium on Computational geometry, pages 150-158, 2005.

[11] P. Indyk and A. Naor. Nearest neighbor preserving embeddings. In ACM Transactions on Algorithms (To appear).

[12] W. B. Johnson, J. Lindenstrauss, and G. Schechtman. Extensions of lipschitz maps into banach spaces. Israel J. Math., 54(2):129-138, 1986.

[13] A. Karzanov. Minimum 0-extensions of graph metrics. European Journal of Combinatorics, 19(1):71-101, 1998.

[14] P. Klein, S. A. Plotkin, and S. B. Rao. Excluded minors, network decomposition, and multicommodity flow. In Proceedings of the 25th ACM Symposium on the Theory of Computing (STOC), pages 682-690, 1993.

[15] G. Konjevod, A. W. Richa, and D. Xia. Optimal-stretch name-independent compact routing in doubling metrics. In The twenty-fifth annual ACM symposium on Principles of distributed computing, 2006.

[16] G. Konjevod, A. W. Richa, and D. Xia. Optimal scale-free compact routing schemes in doubling networks. In Proceedings of the 18th ACM-SIAM Symposium on Discrete Algorithms (SODA), 2007.

[17] R. Krauthgamer and J. R. Lee. The intrinsic dimensionality of graphs. In Proceedings of the thirty-fifth annual ACM symposium on Theory of computing, pages 438-447. ACM Press, 2003.

[18] R. Krauthgamer and J. R. Lee. Navigating nets: simple algorithms for proximity search. In Proceedings of the fifteenth annual ACM-SIAM symposium on Discrete algorithms, pages 798-807. Society for Industrial and Applied Mathematics, 2004.

[19] J. Lee and A. Naor. Absolute lipschitz extendability. Comptes Rendus de l'Acadmie des Sciences - Series I - Mathematics, 338(11):859-862, 2004.

[20] J. Lee and A. Naor and Y. Peres. Trees and Markov convexity. Geometric and Functional Analysis, to appear. Preliminary version in SODA 2006. 
[21] J. Matoušek. On embedding trees into uniformly convex Banach spaces. Israel Journal of Mathematics, 114:221-237, 1999. (Czech version in : Lipschitz distance of metric spaces, C.Sc. degree thesis, Charles University, 1990).

[22] J. Matoušek. Extension of Lipschitz mappings on metric trees. Commentationes Mathematicae Universitatis Carolinae, 31(1):99-104, 1990.

[23] S. B. Rao. Small distortion and volume preserving embeddings for planar and Euclidean metrics. In 15th Annual ACM Symposium on Computational Geometry, pages 300-306, 1999.

[24] S. Semmes. On the nonexistence of bi-Lipschitz parameterizations and geometric problems about $A_{\infty}$-weights. Rev. Mat. Iberoamericana, 12(2):337-410, 1996.

[25] K. Talwar. Bypassing the embedding: Algorithms for low-dimensional metrics. In Proceedings of the 36th ACM Symposium on the Theory of Computing (STOC), pages 281-290, 2004. 